\title{
Anti-basal ganglia antibodies in primary obsessive-compulsive disorder: systematic review and meta-analysis
}

\author{
Daniel M. Pearlman, Haily S. Vora, Brian G. Marquis, Souhel Najjar and Lauren A. Dudley
}

\begin{abstract}
Background
Autoimmune-mediated basal ganglia dysfunction is implicated in the pathophysiology of neuropsychiatric disorders commonly manifesting with obsessive-compulsive features (e.g. Sydenham chorea). The relationship between autoimmunity and primary obsessive-compulsive disorder (OCD), however, is less clear.
\end{abstract}

\begin{abstract}
Aims
To pool data on serum and cerebrospinal fluid (CSF) antibasal ganglia antibody (ABGA) positivity in primary OCD (without neurological or autoimmune comorbidity) relative to controls or neuropsychiatric disorders previously associated with increased odds of ABGA positivity.
\end{abstract}

\section{Method}

We performed electronic database and hand-searches for studies meeting pre-specified eligibility criteria from which we extracted data using a standardised form. We calculated pooled estimates of ABGA positivity using a random-effects model.

\section{Results}

Seven case-control studies totalling 844 participants met the eligibility criteria. Meta-analysis showed that a significantly greater proportion of those with primary OCD were ABGA seropositive compared with various controls (odds ratio
$(\mathrm{OR})=4.97,95 \% \mathrm{Cl} 2.88-8.55, P<0.00001)$. This effect was not associated with heterogeneity or publication bias, and remained significant after stratifying the analysis by age, gender, disease severity, illness duration, immunostaining methodology, study quality, publication type, kind of control group, and sample size. There were no significant differences in ABGA seropositivity for comparisons between primary OCD and Tourette syndrome, attention-deficit hyperactivity disorder or paediatric acute-onset neuropsychiatric syndrome. Results of one study testing CSF samples showed that a significantly greater proportion of participants with primary OCD were ABGA CSF-positive compared with healthy controls $(\mathrm{OR}=5.60,95 \% \mathrm{Cl} 1.04-30.20, P=0.045)$.

\section{Conclusions}

Odds of ABGA seropositivity are increased fivefold in primary OCD compared with controls, but are comparable to those associated with disorders previously associated with ABGA, providing circumstantial evidence of autoimmunity in a subset of those with primary OCD. Further experimental studies are needed to ascertain whether this relationship is causal.

\section{Declaration of interest}

None.
Obsessive-compulsive disorder (OCD) is a severely impairing and often treatment-refractory neuropsychiatric disorder affecting $1-3 \%$ of the population. ${ }^{1-4}$ Symptoms are characterised by recurrent, ego-dystonic, intrusive thoughts (obsessions) and stereotyped thoughts or behaviours (compulsions). ${ }^{5-9}$ Structural and functional aberrations of the corticostriatal thalamocortical (CSTC) circuit are centrally implicated in the pathophysiology of OCD. ${ }^{10-12}$ These include abnormalities involving cerebral glucose metabolism, ${ }^{10,13}$ grey and white matter volume, ${ }^{14}$ functional connectivity ${ }^{15,16}$ and increased glutamatergic tone. ${ }^{17,18}$ Several studies have reported a dose-dependent relationship between treatment-responsiveness in OCD and improvements of these abnormalities. ${ }^{13,19,20}$ Upstream causes of CSTC abnormalities are thought to involve a combination of genetic, ${ }^{21,22}$ glutamatergic $^{17,23}$ and immunological mechanisms. ${ }^{6,7,24-26}$ That both obsessive-compulsive symptomatology and basal ganglia dysfunction are common occurrences among a spectrum of neuropsychiatric disorders with a known (Sydenham chorea ${ }^{27-31}$ ) or suspected (paediatric acute-onset neuropsychiatric syndrome (PANS), ${ }^{27,32,33}$ Tourette syndrome ${ }^{34,35}$ ) autoimmune aetiology, raises the possibility that primary OCD exists along a continuum with these disorders on the basis of overlapping clinical phenomenology and pathophysiological mechanisms respectively. ${ }^{36-41}$ With regard to the latter, these disorders are thought to result from a molecular mimicry mechanism whereby group A $\beta$-hemolytic streptococcal antibodies cross-react with epitopes expressed on the surface of neurons in the basal ganglia, thereby altering neuronal signaling. ${ }^{27,29,42-45}$ Evidence supporting this hypothesis derives from clinical studies showing increased odds of anti-basal ganglia antibody (ABGA) seropositivity in these disorders, ${ }^{27-35,46}$ and experimental studies demonstrating the deposition of ABGA and an inducible neuropsychiatric phenotype in rats following exposure either to group A streptococci or the serum of individuals with Sydenham chorea or PANS. ${ }^{27,47}$ ABGA isolated from such experiments exhibit high avidity for discrete sets of antigens possessing variable degrees of proteomic sequence overlap with streptococcal surface proteins including lysoganglioside GM1, 29,30,43 tubulin, $^{27,28}$ dopamine receptor 1 (DR1) and DR2, ${ }^{27,48}$ and the neuronal glycolytic enzymes: aldolase $C$, neuron-specific gamma- $(\gamma)$ enolase, non- neuronal alpha- $(\alpha)$ enolase and pyruvate kinase $\mathrm{M} 1 .^{35,49}$ Although existing evidence supports the presence of basal ganglia dysfunction in primary OCD and implicates ABGA-mediated basal ganglia dysfunction in neuropsychiatric disorders that frequently manifest with obsessivecompulsive symptoms, the relationship between ABGA and primary OCD remains unclear. We aimed to address this uncertainty by pooling data across studies having evaluated ABGA positivity among individuals with primary OCD relative to various controls or neuropsychiatric disorders previously associated with ABGA.

\section{Method}

Methods used to perform this meta-analysis were devised in accordance with the Preferred Reporting Items for Systematic 
reviews and Meta-Analyses (PRISMA) statement ${ }^{50}$ and the Meta-analysis Of Observational Studies in Epidemiology (MOOSE) guidelines. ${ }^{51}$

\section{Data sources}

We performed a standardised electronic database search for published articles indexed within Ovid MEDLINE (1946-2013), PsycINFO (1806-2013) and/or EMBASE (1974-2013) from inception through to March 2013; there were no search limits or restrictions on language (online Table DS1). To identify unpublished data, we searched BIOSIS previews (1969-2013) and Web of Science (1900-2013), applying search limits for 'conference proceedings' and 'meeting abstracts' to the latter. We supplemented these searches by manually reviewing the reference lists of content-relevant articles.

\section{Study selection}

Eligible studies were delimited to those providing data on ABGA positivity in sera and/or cerebrospinal fluid (CSF) among individuals with primary OCD (cases) compared with one or more comparison groups, to include controls (with any clearly defined inclusion criteria) and/or other neuropsychiatric disorders previously associated with ABGA (henceforth 'ABGA-associated neuropsychiatric disorders'). We required that OCD was diagnosed using a validated approach and that ABGA positivity was assessed using one of the following immunostaining assay techniques: immunohistochemistry, Western blotting, immunocytochemistry, flow cytometry, enzyme-linked immunosorbent assay (ELISA) or immunoprecipitation. We excluded studies for which cases included participants with OCD in the context of ABGA-associated neuropsychiatric disorders in addition to those with primary OCD, unless we were able to extract outcome data separately for participants with primary OCD. We screened titles/abstracts of each unique record identified by our searches to exclude those that were clearly ineligible. We reviewed the full-texts of each remaining record in detail.

\section{Data extraction and synthesis}

After determining our eligible studies, we extracted pre-specified data from each onto a standardised form. Extracted data included characteristics of study sources (eligibility criteria, authors, year of publication, study design, country, publication type, sample size), participants (age, gender, duration of illness, disease severity, diagnostic criteria) and immunostaining assay methodology (techniques, dilutions, tissue preparation). We evaluated study quality using the Newcastle-Ottawa Quality Assessment Scale, ${ }^{52}$ which we adapted to address sources of bias specific to our research question (online Table DS2). Specifically, for each study, we rated the following domains as low, unclear, or high risk of bias: (a) adequate definition of cases; (b) representativeness of cases; (c) adequate definition of controls; (d) comparability of the mean age for case and control groups; (e) comparability of the percentage of females in case and control groups; and (f) masking of outcome assessors to sample case/control status.

The main outcomes were ABGA seropositivity (primary) and ABGA CSF-positivity (secondary), which we defined as specific immunoreactivity (as reported by primary study authors) of sample sera or CSF against basal ganglia homogenate, or when this information was not available, against isolated recombinant forms of one or more putative ABGA antigens (lysoganglioside GM1, tubulin, DR1, DR2, aldolase C, $\gamma / \alpha$-enolase, pyruvate kinase M1). We extracted dichotomous event data on the odds of ABGA positivity in each study group. We summarised these data using odds ratios (ORs) and 95\% confidence interval (CI) point estimates for each comparison of interest (primary OCD $v$. controls; primary OCD $v$. each ABGA-associated neuropsychiatric disorder).

We stipulated immunostaining decision-rules to minimise potential biases related to interstudy variability in immunostaining assay methodology used to assess ABGA positivity. First, for studies using more than one immunostaining assay technique, we preferentially used the results derived from immunohistochemistry. In contrast to Western blotting, immunohistochemistry provides cellular morphological and localisation data and maintains proteins in their natural conformation, which increase the likelihood that any observed immunoreactivity reflects specific neuronal antibody-antigen interactions as they might occur in situ. ${ }^{53}$ Second, for studies using immunohistochemistry that reported zero ABGA positivity events in both cells, we substituted the results from Western blotting. Last, we preferentially used results from Western blotting over ELISA based on evidence that the former has superior sensitivity and specificity for ABGA. ${ }^{42,54}$

Two authors (D.M.P. and H.S.V.) independently assessed study eligibility and performed data extraction. Discrepancies with regard to either process were resolved by consensus. A review author with content expertise in neuropathology (S.N.) reviewed all data extracted on immunostaining methodology for accuracy. We attempted to derive any missing data from studies using indirect calculations and contacted study authors if needed.

\section{Statistical analyses}

All statistical analyses were conducted using the Stata Statistical Software package version 12 for Mac. For each comparison of interest, we pooled point estimate ORs and 95\% CIs of ABGA seropositivity or CSF-positivity into a combined-effect summary estimate using the random-effects model of DerSimonian \& Laird. ${ }^{55}$ We tested for the presence of heterogeneity by calculating Cochrane's $Q$ statistic $(P<0.10$ denotes significant heterogeneity), and quantified any observed heterogeneity by calculating the $I^{2}$ statistic $\left(I^{2} \leqslant 25 \%, \leqslant 50 \%\right.$ and $\leqslant 75 \%$ were considered indicative of low, moderate and high heterogeneity respectively). ${ }^{56}$ We performed a funnel plot test to assess the potential for publication bias. ${ }^{57}$ We performed sensitivity analyses to assess the influence of decisions made during the review process, including: (a) immunostaining method decision-rules; (b) including sources of unpublished data (meeting abstracts, data obtained from study authors); and (c) including studies with unclear or high risk of bias for each risk of bias domain. Study-level characteristics analysed by subgroup analyses were age strata, disease severity, type of control group, immunostaining technique and antigenic sources. Those analysed by random-effects meta-regression analyses ${ }^{58}$ were primary OCD groups' mean age, percent female, Yale-Brown Obsessive Compulsive Scale (Y-BOCS) score s9 $^{59}$ and mean duration of illness.

\section{Results}

Results of our combined search methods and study selection process are presented in Fig. 1 . We excluded 40 records after reviewing their full-texts (online Table DS3 and online supplemental references). Of these, we excluded five potentially eligible studies because they did not provide sufficient data to determine eligibility and attempts to contact the authors for clarifying data were unsuccessful. ${ }^{60-64}$ Seven case-control studies met all eligibility criteria and were included for systematic review and meta-analysis (Table 1)..$^{65-71}$ Of these, six ${ }^{65-70}$ were 
Table 1 Characteristics of participants, immunostaining methods and risk of bias assessment in included studies

\begin{tabular}{|c|c|c|c|c|c|c|c|}
\hline $\begin{array}{l}\text { Category or subgroup } \\
\text { characteristics }\end{array}$ & $\begin{array}{l}\text { Nicholson et al } \\
\qquad(2012)^{65}\end{array}$ & $\begin{array}{l}\text { Gause et al } \\
(2009)^{66}\end{array}$ & $\begin{array}{l}\text { Maina et al } \\
(2008)^{67}\end{array}$ & $\begin{array}{l}\text { Bhattacharyya } \\
\text { et al (2009) }\end{array}$ & $\begin{array}{l}\text { Morer et al } \\
(2009)^{69}\end{array}$ & $\begin{array}{l}\text { Dale et al } \\
(2005)^{70}\end{array}$ & $\begin{array}{l}\text { Parveen et al } \\
(2000)^{71}\end{array}$ \\
\hline Primary OCD, $n(\%)$ & $95(65)$ & $13(15)$ & $72(61)$ & $23(50)$ & $29(40)$ & $33(14)$ & $32(23)$ \\
\hline Age, years: mean (s.d.) & $42(13)$ & $14(3)$ & $35(13)$ & $25(9)$ & $13(3)$ & 13 (NR) & 16 (NR) \\
\hline Female, $n(\%)$ & $51(54)$ & $5(39)$ & $30(42)$ & $5(22)$ & $15(53)$ & $13(40)$ & $17(53)$ \\
\hline Illness duration, years: mean (s.d.) & 26 (NR) & 8 (NR) & 14 (NR) & 5 (NR) & NR (NR) & 4 (NR) & NR (NR) \\
\hline Y-BOCS score, mean (s.d.) & 23 (NR) & 27 (NR) & $25(7)$ & $26(6)$ & $28(6)$ & 20 (NR) & 11 (NR) \\
\hline Controls, $n(\%)$ & $50(34)$ & $29(34)$ & $44(37)$ & $23(50)$ & $19(26)$ & $190(79)$ & $51(37)$ \\
\hline Age, years: mean (s.d.) & $46(13)$ & $12(2)$ & $56(17)$ & $32(13)$ & 13 (3) & 9 (NR) & 15 (NR) \\
\hline Female, $n(\%)$ & $32(64)$ & $17(59)$ & $26(59)$ & $5(22)$ & $9(47)$ & $89(47)$ & $14(28)$ \\
\hline Tourette syndrome, a $n$ (\%) & $1(1)$ & $23(27)$ & $2(2)$ & $0(0)$ & $16(22)$ & 8 (3) & $38(28)$ \\
\hline Age, years: mean (s.d.) & NR (NR) & $13(4)$ & NR (NR) & - & $12(2)$ & NR (NR) & 13 (NR) \\
\hline Female, $n(\%)$ & NR (NR) & $7(30)$ & NR (NR) & - & $3(19)$ & NR (NR) & $8(21)$ \\
\hline ADHD, $n(\%)$ & $0(0)$ & $0(0)$ & $0(0)$ & $0(0)$ & $0(0)$ & 9 (4) & $16(12)$ \\
\hline Age, years: mean (s.d.) & - & - & - & - & - & NR (NR) & NR (NR) \\
\hline Female, $n(\%)$ & - & - & - & - & - & NR (NR) & NR (NR) \\
\hline PANS, $n(\%)$ & $0(0)$ & $20(24)$ & $0(0)$ & $0(0)$ & $8(11)$ & $0(0)$ & $0(0)$ \\
\hline Age, years: mean (s.d.) & - & $11(2)$ & - & - & NR (NR) & - & - \\
\hline Female, $n(\%)$ & - & $8(40)$ & - & - & NR (NR) & - & - \\
\hline \multicolumn{8}{|l|}{ Immunostaining methods } \\
\hline Technique $^{\mathrm{b}}$ & Western blot & $\mathrm{IHC}$ & IHC & Western blot & Western blot & Western blot & $\mathrm{IHC}$ \\
\hline $\begin{array}{l}\text { Homogenate } \\
\text { Isolate antigens }\end{array}$ & $\begin{array}{c}- \\
\text { NGES }\end{array}$ & Globus pallidus & $\begin{array}{c}\text { Basal ganglia }^{d} \\
-\end{array}$ & $\begin{array}{c}\text { Basal ganglia }{ }^{e} \\
\ldots\end{array}$ & $\begin{array}{c}\text { Putamen } \\
-\end{array}$ & $\begin{array}{c}\text { Caudate, putamen } \\
\text { _ }\end{array}$ & Globus pallidus \\
\hline Source & Recombinant & Human brain & Rat brain & Human brain & Human brain & Human brain & Rat brain \\
\hline Dilution, sample sera & $1: 300$ & $1: 50$ & $1: 100$ & 1:250 & 1:1000 & $1: 300$ & $1: 10^{c}$ \\
\hline Dilution, secondary antibody & NR & 1:40 & NR & 1:3000 & 1:20 000 & 1:1000 & NR \\
\hline Negative control (IHC only) & - & Yes & Yes & - & - & - & Yes \\
\hline Detection (Western blot only) & $\mathrm{ECL}$ & - & - & Colorimetric & Colorimetric & ECL & - \\
\hline \multicolumn{8}{|l|}{ Risk of bias assessment } \\
\hline Case definition & Low & Low & Low & Low & Low & Low & High \\
\hline Case representativeness & Low & High & Unclear & High & High & Unclear & High \\
\hline Control definition & Low & Low & Unclear & Low & Low & High & Low \\
\hline Age-matched & Low & Low & High & High & Low & High & High \\
\hline Gender-matched & Low & High & High & Low & Low & Low & High \\
\hline Masked outcome assessment & Low & Low & Unclear & Low & Unclear & Unclear & Low \\
\hline \multicolumn{8}{|c|}{$\begin{array}{l}\text { ADHD, attention-deficit hyperactivity disorder; ECL, Electrochemiluminescence; IHC, immunohistochemistry; NGES, neuronal glycolytic enzymes; NR, not reported; OCD, obsessive- } \\
\text { compulsive disorder; PANS, paediatric acute-onset neuropsychiatric syndrome; s.d., standard deviation; Y-BOCS, Yale-Brown Obsessive Compulsive Scale. } \\
\text { a. Refers to baseline characteristics data that was provided in aggregate for Tourette syndrome and primary OCD participants. } \\
\text { b. Refers to the immunostaining method that yielded the outcome data included in our meta-analysis. } \\
\text { c. Refers to starting concentration, which was titrated to an endpoint of reactivity at maximum dilution for each sample using two-fold serial dilutions. } \\
\text { d. Not totherwise specified. } \\
\text { e. Caudate, putamen, and globus pallidus. }\end{array}$} \\
\hline
\end{tabular}

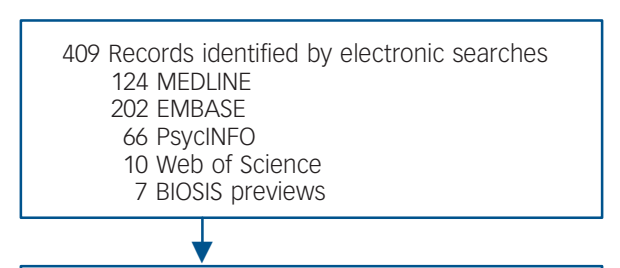

238 Titles and abstracts screened after removing duplicates

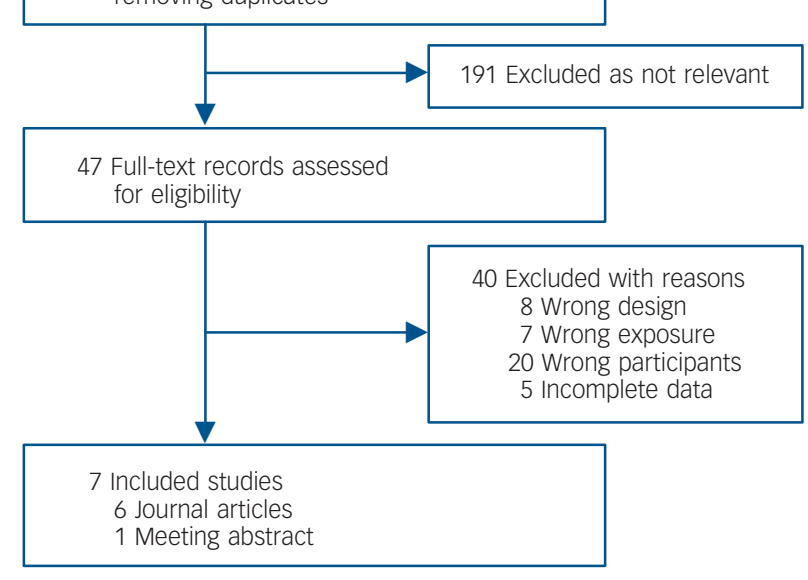

Fig. 1 Study attrition diagram. peer-reviewed journal articles and one ${ }^{71}$ was a meeting abstract. We extracted outcome data directly from these sources with two exceptions, ${ }^{68,71}$ which necessitated contacting the study authors; one $^{68}$ because the original report only provided data on anti-basal ganglia/thalamic antibody seropositivity in aggregate rather than on ABGA alone, and the other ${ }^{71}$ because it was a meeting abstract that provided insufficient detail. Antigenic substrates used to assess ABGA seropositivity varied from homogenate consisting of putamen alone, caudate alone, or combined caudate, putamen and globus pallidus, to each of the neuronal glycolytic enzymes obtained as commercial recombinant isolated antigens. Primary outcome data were based on immunohistochemistry in three studies, ${ }^{66,67,71}$ and on Western blotting in four; ${ }^{65,68-70}$ of the latter group, one study that also utilised immunohistochemistry reported zero events of ABGA seropositivity in both cells. ${ }^{69}$ Immunostaining protocols provided variable sample sera and secondary antibody dilutions, immunoreactivity detection techniques and tissue preparation. All studies utilised samples consisting of whole serum rather than isolated immunoglobulin.

Studies included 844 total participants, consisting of 297 with primary OCD, 406 controls and 141 with ABGA-associated neuropsychiatric disorders. Primary OCD cohorts included children, ${ }^{66,69,70}$ adults $^{65,67}$ or both. ${ }^{68,71}$ Percent female ranged from 22 to 54. Mean duration of illness ranged from 4 to 26 years. Mean Y-BOCS scores ranged from 11 (mild) to 28 (severe). Control participants were divided into four mutually exclusive 


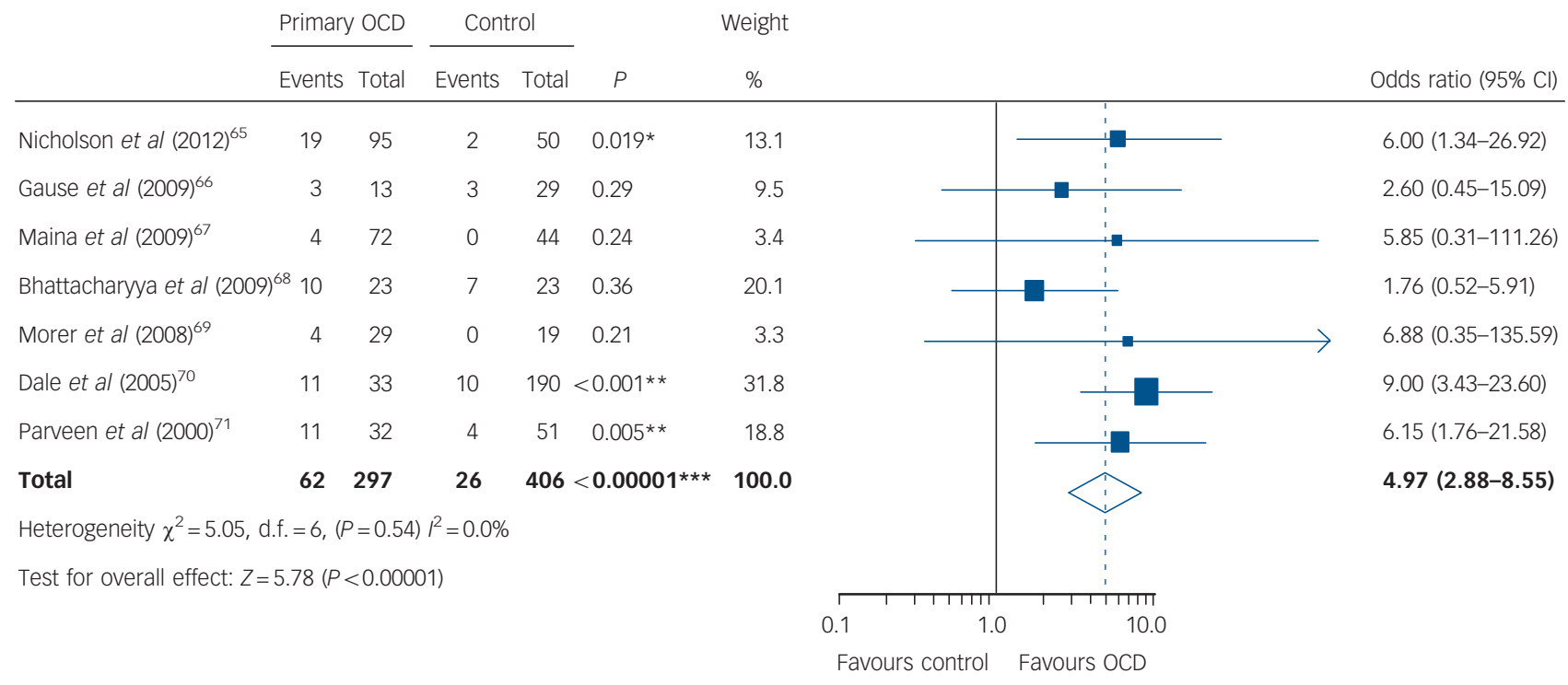

Fig. 2 Forest plot of anti-basal ganglia antibody seropositivity in primary obsessive-compulsive disorder (OCD) compared with controls.

subgroups: healthy $(n=162),{ }^{66,68-71}$ psychiatric $(n=94),{ }^{65,67}$ neurological $(n=100)^{70}$ or autoimmune $(n=50){ }^{70}$ Psychiatric controls consisted of individuals with major depressive disorder, ${ }^{65,67}$ bipolar disorder type $\mathrm{I},{ }^{67}$ bipolar disorder type II, ${ }^{67}$ and schizophrenia. ${ }^{65,67}$ Neurological controls consisted of individuals with stroke, metabolic movement disorders, and encephalitis. ${ }^{70}$ Autoimmune controls consisted of individuals with rheumatic carditis, glomerulonephritis and other autoimmune diseases that explicitly did not have neuropsychiatric manifestations. ${ }^{70}$ ABGA-associated neuropsychiatric disorders identified among included studies consisted of Tourette syndrome $(n=88),{ }^{65-67,69-71}$ attention-deficit hyperactivity disorder (ADHD) $(n=25)^{70,71}$ and PANS $(n=28) .{ }^{66,69}$

All seven studies provided data on ABGA seropositivity for 297 participants with primary OCD compared with 406 controls, yielding a total sample of 703 participants. ${ }^{65-71}$ Individual study ORs favoured primary OCD in all seven studies, of which this difference of proportions constituted a statistically significant

Table 2 Sensitivity and subgroup analyses for anti-basal ganglia antibody (ABGA) seropositivity in primary obsessive-compulsive disorder (OCD) compared with controls

\begin{tabular}{|c|c|c|c|c|c|c|c|}
\hline \multirow[b]{2}{*}{ Analysis type } & \multicolumn{2}{|c|}{ Sample } & \multicolumn{2}{|c|}{ ABGA seropositivity } & \multicolumn{3}{|c|}{ Heterogeneity } \\
\hline & Studies & $n$ & OR $(95 \% \mathrm{Cl})$ & $P$ & $\chi^{2}$ & $P$ & $1^{2}$ \\
\hline \multicolumn{8}{|l|}{ Sensitivity } \\
\hline Immunostaining decision-rules ${ }^{65,66,68-71}$ & 7 & 800 & $3.12(1.89-5.14)$ & $<0.001^{* * *}$ & 5.29 & 0.508 & 0.0 \\
\hline Excluding unpublished data $65,66,69,70$ & 5 & 574 & $6.54(3.26-13.10)$ & $<0.001^{* * *}$ & 4.91 & 0.427 & 0.0 \\
\hline Case definition ${ }^{65,66,68-70, a}$ & 6 & 620 & $4.73(2.59-8.63)$ & $<0.001^{* * *}$ & 4.91 & 0.427 & 0.0 \\
\hline Case representativeness ${ }^{65, a}$ & 1 & 145 & $6.00(1.34-26.92)$ & $0.019^{*}$ & - & - & - \\
\hline Control definition ${ }^{74,75,77,78,80, a}$ & 5 & 587 & $3.68(1.87-7.22)$ & $<0.001^{* * *}$ & 2.83 & 0.586 & 0.0 \\
\hline Age-matched $65,66,69, a$ & 3 & 235 & $4.49(1.55-13.04)$ & $0.006^{* *}$ & 0.62 & 0.734 & 0.0 \\
\hline Gender-matched $d^{74,77-79, a}$ & 4 & 462 & $4.89(2.08-11.50)$ & $<0.001^{\star * *}$ & 4.42 & 0.220 & 32.1 \\
\hline Masked outcome assessment ${ }^{74,75,77,80, a}$ & 4 & 386 & $3.56(1.78-7.11)$ & $<0.001^{* * *}$ & 2.64 & 0.450 & 0.0 \\
\hline \multicolumn{8}{|l|}{ Subgroup } \\
\hline Paediatric, ages $<18$ years $66,69,70$ & 3 & 397 & $6.77(3.00-15.26)$ & $<0.001^{* \star *}$ & 1.49 & 0.474 & 0.0 \\
\hline Adult, ages $\geqslant 18$ years $^{74,76}$ & 2 & 167 & $5.97(1.57-22.74)$ & $<0.001^{* * *}$ & 0.00 & 0.988 & 0.0 \\
\hline Paediatric and adult, all ages ${ }^{77,80}$ & 2 & 55 & $3.25(0.95-11.11)$ & 0.06 & 1.98 & 0.159 & 49.6 \\
\hline \multicolumn{8}{|l|}{ Disorder severity } \\
\hline Mild to moderate, $Y$-BOCS $<24^{65,70,71}$ & 3 & 451 & $7.40(3.75-14.63)$ & $<0.001^{* * *}$ & 0.34 & 0.844 & 0.0 \\
\hline Severe to extreme, Y-BOCS $\geqslant 24^{66-69}$ & 4 & 393 & $2.47(1.00-6.08)$ & $0.049^{*}$ & 1.13 & 0.770 & 0.0 \\
\hline \multicolumn{8}{|l|}{ Control type } \\
\hline Healthy ${ }^{75,77-80}$ & 5 & 459 & $4.14(1.83-9.31)$ & $0.001 * *$ & 4.85 & 0.303 & 17.6 \\
\hline Autoimmune disorders ${ }^{70}$ & 1 & 133 & $4.50(1.39-14.55)$ & $0.012^{*}$ & - & - & - \\
\hline Neurological disorders ${ }^{70}$ & 1 & 83 & $12.00(3.49-41.24)$ & $<0.001^{* * *}$ & - & - & - \\
\hline Psychiatric disorders ${ }^{65,67}$ & 2 & 261 & $5.97(1.57-22.74)$ & $0.009^{* *}$ & 0.00 & 0.988 & 0.0 \\
\hline \multicolumn{8}{|l|}{ Immunostaining assay } \\
\hline Immunohistochemistry ${ }^{75,56,80}$ & 3 & 340 & $4.72(1.80-12.40)$ & $0.002 * *$ & 0.64 & 0.727 & 0.0 \\
\hline Western blotting $74,77-79$ & 4 & 504 & $4.89(2.08-11.50)$ & $<0.001^{* * *}$ & 4.42 & 0.220 & 32.1 \\
\hline \multicolumn{8}{|l|}{ Antigenic substrate } \\
\hline Human source ${ }^{66,68-70}$ & 4 & 443 & $4.08(1.60-10.42)$ & $0.003^{* *}$ & 4.82 & 0.186 & 37.7 \\
\hline Non-human source 67,71 & 2 & 255 & $6.11(1.93-19.37)$ & $0.002^{* *}$ & 0.00 & 0.975 & 0.0 \\
\hline
\end{tabular}




\begin{tabular}{|c|c|c|c|c|c|c|}
\hline & \multicolumn{2}{|c|}{ Sample } & \multicolumn{2}{|c|}{ ABGA seropositivity } & \multicolumn{2}{|c|}{ Heterogeneity } \\
\hline & Studies & $n$ & $\mathrm{~B}(95 \% \mathrm{Cl})$ & $P$ & $\chi^{2}$ & $P$ \\
\hline Sample, number of participants ${ }^{74,75,77-80}$ & 7 & 844 & $0.01(-0.00$ to 0.02$)$ & 0.156 & 1.26 & 0.939 \\
\hline \multicolumn{7}{|l|}{ Primary OCD group } \\
\hline Age, years: mean ${ }^{65,66,68-71}$ & 7 & 297 & $-0.01(-0.08$ to 0.07$)$ & 0.773 & 3.90 & 0.564 \\
\hline Female, $\%^{74,75,77-80}$ & 7 & 297 & $3.86(-2.45$ to 10.18$)$ & 0.177 & 1.57 & 0.905 \\
\hline Y-BOCS score, mean $65,66,68-71$ & 7 & 297 & $-0.06(-0.19$ to 0.07$)$ & 0.282 & 2.59 & 0.763 \\
\hline Illness duration, years: mean ${ }^{65-68,70}$ & 7 & 297 & $0.02(-0.14$ to 0.18$)$ & 0.691 & 3.52 & 0.315 \\
\hline \multicolumn{7}{|l|}{ Control group } \\
\hline Age, years: mean ${ }^{65-68,70}$ & 7 & 406 & $-0.01(-0.16$ to 0.04$)$ & 0.544 & 3.62 & 0.605 \\
\hline Female, $\%^{65-68,70}$ & 7 & 206 & $0.02(-0.03$ to 0.06$)$ & 0.398 & 3.19 & 0.671 \\
\hline
\end{tabular}

(a)

\begin{tabular}{|c|c|c|c|c|}
\hline & \multicolumn{2}{|c|}{ Primary OCD } & \multicolumn{2}{|c|}{ Tourette syndrome } \\
\hline & Events & Total & Events & Total \\
\hline Nicholson et al (2012) ${ }^{65}$ & 19 & 95 & 0 & 1 \\
\hline Gause et al (2009) $)^{66}$ & 3 & 13 & 6 & 23 \\
\hline Maina et al $(2009)^{67}$ & 4 & 72 & 0 & 2 \\
\hline Morer et al $(2008)^{69}$ & 4 & 29 & 1 & 16 \\
\hline Dale et al $(2005)^{70}$ & 11 & 33 & 6 & 8 \\
\hline Parveen et al $(2000)^{71}$ & 11 & 32 & 14 & 38 \\
\hline TO & 52 & 274 & 27 & 88 \\
\hline
\end{tabular}

Heterogeneity $\chi^{2}=4.20$, d.f. $=5,(P=0.52) l^{2}=0.0 \%$

Test for overall effect: $Z=0.97(P=0.33)$

$\%$

Odds ratio $(95 \% \mathrm{Cl})$

4.5

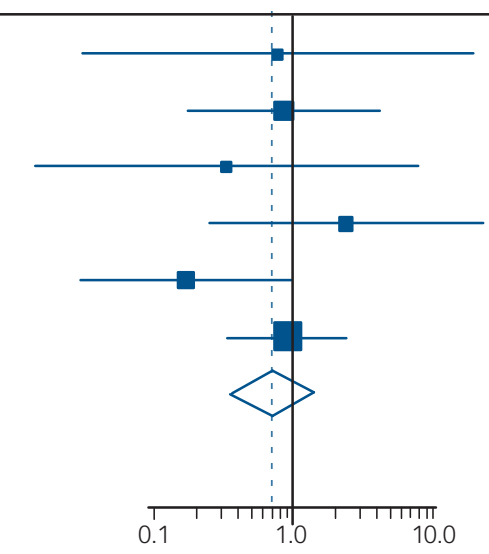

$0.76(0.03-19.51)$

$0.85(0.17-4.17)$

$0.33(0.01-7.92)$

$2.40(0.24-23.53)$

$0.17(0.03-0.97)$

$0.90(0.34-2.40)$

$0.71(0.36-1.41)$

(b)

Favours Tourette Favours OCD

Odds ratio $(95 \% \mathrm{Cl})$

\begin{tabular}{llllll}
\hline Dale et al $(2005)^{70}$ & 11 & 33 & 4 & 9 & 42.3 \\
Parveen et al $(2000)^{71}$ & 11 & 32 & 5 & 16 & 57.7 \\
Total & $\mathbf{2 2}$ & $\mathbf{6 5}$ & $\mathbf{9}$ & $\mathbf{2 5}$ & $\mathbf{1 0 0 . 0}$
\end{tabular}

Heterogeneity $\chi^{2}=0.37$, d.f. $=1,(P=0.54) P^{2}=0.0 \%$

Test for overall effect: $Z=0.23(P=0.81)$

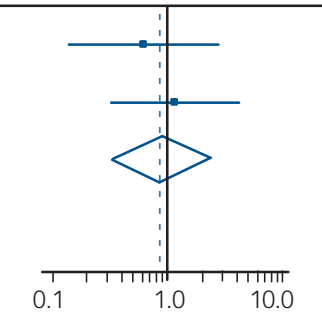

$0.63(0.14-2.80)$

$1.15(0.32-4.16)$

$0.89(0.34-2.36)$

Favours ADHD Favours OCD

(c)

\begin{tabular}{|c|c|c|c|c|c|}
\hline & \multicolumn{2}{|c|}{ Primary OCD } & \multicolumn{2}{|c|}{ PANS } & Weight \\
\hline & Events & Total & Events & Total & $\%$ \\
\hline Gause et al (2009) & 3 & 13 & 4 & 20 & \\
\hline Morer et al (2008) $)^{69}$ & 4 & 29 & 2 & 8 & \\
\hline Total & 7 & 42 & 6 & 28 & \\
\hline
\end{tabular}

Heterogeneity $\chi^{2}=0.49$, d.f. $=1,(P=0.48) P^{2}=0.0 \%$

Test for overall effect: $Z=0.34(P=0.74)$

Odds ratio $(95 \% \mathrm{Cl})$

$1.20(0.22-6.52)$

$0.48(0.07-3.26)$

$0.80(0.23-2.86)$

Fig. 3 Forest plot of anti-basal ganglia antibody seropositivity in primary obsessive-compulsive disorder (OCD) compared with

(a) Tourette syndrome, (b) attention-deficit hyperactivity disorder (ADHD), and (c) paediatric acute-onset neuropsychiatric syndrome (PANS). 
difference in three studies. ${ }^{65,70,71}$ Pooled analyses showed that a significantly greater proportion of participants with primary OCD were ABGA seropositive compared with various controls ( $\mathrm{OR}=4.97,95 \%$ CI 2.88-8.55, $P<0.00001$; Fig. 2), without evidence of heterogeneity $\left(\chi^{2}=5.05, P=0.54, I^{2}=0.0 \%\right)$ or publication bias (online Fig. DS1). Results of sensitivity analyses indicated that the direction, magnitude and statistical significance of this effect were independent of publication type, immunostaining methods and inclusion of studies with an unclear or high risk of bias for each of the domains assessed (Table 2). This effect also remained statistically significant for each subgroup analysed with the exception of studies involving both paediatric and adult participants. Random-effects meta-regression analyses showed no significant differences between the magnitude of individual study ORs and any of the study-level characteristics analysed (Table 3). Results from the two studies ${ }^{65,70}$ relating ABGA seropositivity to participant-level data showed no statistically significant differences between ABGA seropositive and ABGA seronegative participants with regard to age, gender or duration of illness; of these, one study ${ }^{70}$ documented significantly worse disease severity (i.e. mean Y-BOCS scores) among ABGA seropositive participants. Of the seven included studies, one ${ }^{68}$ provided data on ABGA CSFpositivity, yielding a total sample of 46 participants (23 with primary OCD, 23 healthy controls). A significantly greater proportion of those with primary OCD were ABGA CSF-positive compared with healthy controls $(\mathrm{OR}=5.60,95 \%$ CI $1.04-30.20$, $P=0.045)$.

Two studies ${ }^{65,66}$ provided data on immunoreactivity against the isolated antigens aldolase $\mathrm{C}, \gamma$-enolase and pyruvate kinase M1 (obtained as commercial recombinant antigens). Of these, the data from one ${ }^{65}$ contributed to the above-reported summary estimate for ABGA seropositivity in primary OCD compared with controls. Data from the other study did not because primary outcome data were also provided based on the results of an assay involving basal ganglia homogenate. A significantly greater proportion of participants with primary OCD were seropositive for $\gamma$-enolase antibodies compared with controls $(\mathrm{OR}=8.12$, 95\% CI 1.72-38.47), but not for aldolase C $(\mathrm{OR}=2.31,95 \% \mathrm{CI}$ $0.61-8.78)$ or pyruvate kinase $\mathrm{M} 1(\mathrm{OR}=0.62,95 \%$ CI $0.21-$ 1.88 ) antibodies. None of the included studies provided data on ABGA positivity, in either sera or CSF, based on assays utilising isolated forms of other putative antigens. In several instances, peak band intensities for primary OCD groups, determined from ABGA seropositive samples by Western blotting using basal ganglia homogenate rather than using isolated antigens, corresponded to putative ABGA antigens $( \pm 2 \mathrm{kDa})$. These included aldolase C $(40 \mathrm{kDa}),{ }^{66,69} \gamma / \alpha$-enolase doublet $(45 \mathrm{kDa}),{ }^{65,66,68,69} \gamma$ - $\alpha$-enolase heterodimer $(98 \mathrm{kDa}),{ }^{66,68,70}$ tubulin $(55 \mathrm{kDa})^{69}$ and pyruvate kinase M1 $(60 \mathrm{kDa}){ }^{65,67,70}$

Six studies provided data on ABGA seropositivity in primary OCD compared with Tourette syndrome, yielding a total sample of 363 (274 with primary OCD, 88 with Tourette syndrome). ${ }^{65-67,69-71}$ The summary estimate was not statistically significant $(\mathrm{OR}=0.71$, 95\% CI 0.36-1.41, $P=0.33$; Fig. 3(a)), without evidence of heterogeneity $\left(\chi^{2}=4.20, P=0.52, I^{2}=0.0 \%\right)$. Two studies ${ }^{70,71}$ provided data on ABGA seropositivity in primary OCD compared with ADHD, yielding a total sample of 90 participants ( 65 with primary OCD, 25 with ADHD). The summary estimate was not statistically significant $(\mathrm{OR}=0.89,95 \%$ CI $0.34-2.36, P=0.81$; Fig. 3(b)), without evidence of heterogeneity $\left(\chi^{2}=0.37 ; \quad P=0.54\right.$; $\left.I^{2}=0.0 \%\right)$. Two studies ${ }^{66,69}$ provided data on ABGA seropositivity in primary OCD compared with PANS, yielding a total sample of 70 participants (42 with primary OCD, 28 with PANS). The summary estimate was not statistically significant $(\mathrm{OR}=0.80$, 95\% CI 0.23-2.86, $P=0.74$; Fig. 3(c)), without evidence of heterogeneity $\left(\chi^{2}=0.49, P=0.48, I^{2}=0.0 \%\right)$. No studies provided data on ABGA CSF-positivity for participants with ABGA-associated neuropsychiatric disorders.

\section{Discussion}

To our knowledge, this is the first systematic review and metaanalysis to assess the relationship between autoimmunity and primary OCD by pooling data from studies having assessed sera and/or CSF samples for ABGA positivity. Our results show that ABGAs are five times more likely to be detected in the serum of individuals with primary OCD compared with control groups consisting of healthy controls as well as those with various psychiatric, neurological and autoimmune disorders. This effect is statistically significant, generalisable to both paediatric and adult forms of primary OCD, and robust to multiple pre-selected sensitivity analyses. This effect derives from seven studies, only three of which reported a statistically significant effect individually, yet all of which reported a consistent effect size as evidenced by the absence of heterogeneity associated with the summary estimates. This suggests that the discordant findings among point estimates of ABGA seropositivity in the extant literature are largely attributable to three inadequately powered studies with Type II error.

In addition, that (a) both studies having assessed immunoreactivity against isolated antigens found significantly increased odds of $\gamma$-enolase antibody, but not aldolase C antibody, seropositivity in primary OCD compared with controls, and (b) four studies identified primary OCD group peak bands corresponding to the $\gamma / \alpha$-enolase doublet, supports the specific nature of the observed association between primary OCD and autoimmunity. Supporting this interpretation, of the neuronal glycolytic enzymes, $\gamma$-enolase exhibits the greatest degree of proteomic sequence overlap with streptococcal pyogenes surface proteins $\left(49 \%\right.$, expected chance identity $\left.=1.0 \times 10^{-109}\right) .{ }^{49}$ Arguing against this interpretation, however, despite the fact that pyruvate kinase M1 also exhibits considerable homology (38\% proteomic sequence overlap) we observed no significant difference in the odds of seropositivity in primary OCD compared with controls in studies utilising recombinant pyruvate kinase as the isolate test antigen. Whether the latter finding is a consequence of Type II error is unknown. Last, existing evidence indicates that those with primary OCD are associated with 5.6-fold increased odds of ABGA CSF-positivity compared with healthy controls. If replicated, these data also favour the hypothesis that primary OCD is autoimmune-mediated in some patients.

\section{Limitations}

Results of this meta-analysis should be interpreted in light of several limitations. First, although sensitivity, subgroup and meta-regression analyses indicated that the observed effect of increased odds of ABGA seropositivity in primary OCD compared with controls remains robust to numerous conditionals, the possibility that residual confounding occurred cannot be excluded. Potential confounders that we did not assess include history of streptococcal exposure, autoimmune disease, immunotherapy and psychotropic medication. Second, interstudy variability in the sensitivity and specificity of immunostaining assays due to methodological heterogeneity limits the validity of inferences regarding the absolute odds of ABGA positivity in each study group. Even if this were definitively shown to be the case, however, it would not necessarily affect the ORs relating the proportions of ABGA-positive participants in each group. Last, that none of the eligible studies tested for seropositivity using 
isolated forms of other putative ABGA antigens (lysoganglioside GM1, tubulin, DR1 or DR2) restricts our ability to comment directly on their relationship with primary OCD. Since antibodies directed against these antigens are known to induce frank chorea in Sydenham chorea, ${ }^{27-30}$ our observation that only one included study identified a primary OCD group peak band corresponding to one of these antigens (tubulin, $55 \mathrm{kDa})^{69}$ may reflect the absence of overt movement sequelae in primary OCD.

\section{Implications}

According to Witebsky's postulates of criteria defining autoimmunity, our results constitute circumstantial evidence of autoimmunity in a subset of primary OCD..$^{72,73}$ To determine whether ABGA are pathogenic, epiphenomena or biomarkers of concomitant autoimmunity secondary to basal ganglia injury (antigen spreading), requires pre-clinical experimental studies designed to provide indirect or direct evidence of autoimmunity. ${ }^{72,73}$ Such studies might be modelled after those supporting the pathogenicity of ABGA in PANS and Sydenham chorea. ${ }^{27,29,30,43}$ Implicit in the design of these experimental studies is an analysis of which, if any, specific epitopes are pathogenic, and whether ABGA-antigen interactions influence signal transduction. Future studies should evaluate the relationship between ABGA positivity and established abnormalities of the CSTS, ${ }^{14}$ glutamatergic tone, ${ }^{17}$ and other genetic abnormalities among those with primary OCD. ${ }^{24,74-76}$ Such studies are likely to clarify whether these abnormalities - largely derived from separate lines of investigation - reflect convergent mechanisms in the pathogenesis of OCD.

The present review also highlights the absence of a standardised immunostaining assay protocol for assessing ABGA seropositivity. Evidenced-based methods that should inform the design of future studies addressing this research question, include the utilisation of:

(a) positive and negative laboratory controls ${ }^{66,67}$

(b) fresh $v$. frozen tissue preparation ${ }^{77}$

(c) serial titration of sample dilution to optimise the signal to noise ratio $^{78}$

(d) isolated immunoglobulin $v$. whole serum ${ }^{77}$

(e) electrochemiluminescence $v$. colorimetric detection ${ }^{77}$

(f) isolated recombinant forms of each putative ABGA autoantigen, and homogenate comprising other cortical regions apart from basal ganglia that are implicated in OCD and which have been associated with ABGA (e.g. thalamic nuclei). ${ }^{68}$

Future studies should also evaluate the effect of duration of illness and disease state severity on the likelihood of ABGA positivity, based on prior studies showing these factors influence the pre-test odds of immunoreactivity. ${ }^{27,79-81}$ In addition, the association between primary OCD and autoimmunity indicated by our results lends support for the rationale to explore the efficacy of rationally based immunomodulatory and anti-inflammatory agents as potential pharmacological treatment approaches; in so doing, clinicians and researchers should aim to identify the subsets of patients for which these approaches would be most appropriate.

Odds of ABGA seropositivity are increased fivefold in primary OCD compared with controls, but are comparable to those associated with disorders previously associated with ABGA, providing circumstantial evidence of autoimmunity in a subset of those with primary OCD. Further experimental studies are needed to ascertain whether this relationship is causal.

\section{Acknowledgements}

We thank Drs Bhattacharyya and Morshed for providing us with their data, and thanks to Drs Robin Larson, Natalie Riblet, Kenneth Roberts, Madhavi Lakkaraja, Richard Ferrell and Jeremiah Brown, for their advice concerning systematic review methods as well as how to improve the manuscript. The authors report no biomedical financial interest or potential conflicts of interest.

Daniel M. Pearlman, MPH, The Dartmouth Institute for Health Policy and Clinical Practice, Geisel School of Medicine at Dartmouth, Lebanon, New Hampshire, and Neuroinflammation Research Group, Epilepsy Center Division, Department of Neurology, NYU School of Medicine, New York; Haily S. Vora, MPH, Brian G. Marquis, MS,The Dartmouth Institute for Health Policy and Clinical Practice, Geisel School of Medicine at Dartmouth, Lebanon, New Hampshire; Souhel Najjar, MD, Neuroinflammation Research Group, Epilepsy Center Division, Department of Neurology, NYU School of Medicine, New York; Lauren A. Dudley, MD, The Dartmouth Institute for Health Policy and Clinical Practice, Geisel School of Medicine at Dartmouth, and Section of Rheumatology, Department of Medicine, DartmouthHitchcock Medical Center, Lebanon, New Hampshire, USA

Correspondence: Daniel M. Pearlman, MPH, Neuroinflammation Research Group, Epilepsy Center Division, Department of Neurology, NYU School of Medicine, 223 East 34th Street, New York, NY 10016, USA. Email: daniel.m.pearlman@gmail.com

First received 15 Aug 2013, final revision 16 Jan 2014, accepted 10 Feb 2014

\section{References}

1 Mancebo MC, Greenberg B, Grant JE, Pinto A, Eisen JL, Dyck I, et al. Correlates of occupational disability in a clinical sample of obsessivecompulsive disorder. Compr Psychiatry 2008; 49: 43-50.

2 Eisen JL, Mancebo MA, Pinto A, Coles ME, Pagano ME, Stout R, et al. Impact of obsessive-compulsive disorder on quality of life. Compr Psychiatry 2006; 47: 270-5.

3 Ayuso-Mateos J. Global Burden of Obsessive-compulsive Disorder in the Year 2000. World Health Organization, 2006.

4 Ruscio AM, Stein DJ, Chiu WT, Kessler RC. The epidemiology of obsessivecompulsive disorder in the National Comorbidity Survey Replication. $\mathrm{Mol}$ Psychiatry 2010; 15: 53-63.

5 Williams MT, Farris SG, Turkheimer E, Pinto A, Ozanick K, Franklin ME, et al. Myth of the pure obsessional type in obsessive-compulsive disorder. Depress Anxiety 2011; 28: 495-500.

6 Abramowitz JS, Taylor S, McKay D. Obsessive-compulsive disorder. Lancet 2009; 374: 491-9.

7 Stein DJ. Obsessive-compulsive disorder. Lancet 2002; 360: 397-405.

8 Rachman S. A cognitive theory of obsessions: elaborations. Behav Res Ther 1998; 36: 385-401

9 Rachman S. A cognitive theory of obsessions. Behav Res Ther 1997; 35 793-802.

10 Maia TV, Cooney RE, Peterson BS. The neural bases of obsessive-compulsive disorder in children and adults. Dev Psychopathol 2008; 20: 1251-83.

11 Welter ML, Burbaud P, Fernandez-Vidal S, Bardinet E, Coste J, Piallat B, et al. Basal ganglia dysfunction in OCD: subthalamic neuronal activity correlates with symptoms severity and predicts high-frequency stimulation efficacy. Transl Psychiatry 2011; 1: e5.

12 Modell JG, Mountz JM, Curtis GC, Greden JF. Neurophysiologic dysfunction in basal ganglia/limbic striatal and thalamocortical circuits as a pathogenetic mechanism of obsessive-compulsive disorder. J Neuropsychiatry Clin Neurosci 1989; 1: 27-36.

13 Baxter LR, Schwartz JM, Bergman KS, Szuba MP, Guze BH, Mazziotta JC, et al. Caudate glucose metabolic rate changes with both drug and behaviour therapy for obsessive-compulsive disorder. Arch Gen Psychiatry 1992; 49: 681-9.

14 Radua J, van den Heuvel OA, Surguladze S, Mataix-Cols D. Meta-analytica comparison of voxel-based morphometry studies in obsessive-compulsive disorder vs other anxiety disorders. Arch Gen Psychiatry 2010; 67: 701-11.

15 Chamberlain SR, Menzies L, Hampshire A, Suckling J, Fineberg NA, de Campo N, et al. Orbitofrontal dysfunction in patients with obsessivecompulsive disorder and their unaffected relatives. Science 2008; 321 421-2.

16 Harrison BJ, Soriano-Mas C, Pujol J, Ortiz H, Lopez-Sola M, Hernandez-Ribas $\mathrm{R}$, et al. Altered corticostriatal functional connectivity in obsessivecompulsive disorder. Arch Gen Psychiatry 2009; 66: 1189-200. 
17 Pittenger C, Bloch MH, Williams K. Glutamate abnormalities in obsessive compulsive disorder: neurobiology, pathophysiology, and treatment. Pharmacol Ther 2011; 132: 314-32.

18 Chakrabarty K, Bhattacharyya S, Christopher R, Khanna S. Glutamatergic dysfunction in OCD. Neuropsychopharmacology 2005; 30: 1735-40.

19 Rosenberg DR, Benazon NR, Gilbert A, Sullivan A, Moore GJ. Thalamic volume in pediatric obsessive-compulsive disorder patients before and after cognitive behavioural therapy. Biol Psychiatry 2000; 48: 294-300.

20 Hansen ES, Hasselbalch S, Law I, Bolwig TG. The caudate nucleus in obsessive-compulsive disorder. Reduced metabolism following treatment with paroxetine: a PET study. Int I Neuropsychopharmacol 2002; 5: 1-10.

21 Stewart SE, Mayerfeld C, Arnold PD, Crane AJ, O'Dushlaine C, Fagerness JA et al. Meta-analysis of association between obsessive-compulsive disorder and the $3^{\prime}$ region of neuronal glutamate transporter gene SLC1A1. Am J Med Genet B Neuropsychiatr Genet 2013; 162B: 367-79.

22 Stewart SE, YU D, Scharf JM, Neale BM, Fagerness JA, Mathews CA, et al. Genome-wide association study of obsessive-compulsive disorder. $\mathrm{Mol}$ Psychiatry 2013; 18: 788-98.

23 Wu K, Hanna GL, Rosenberg DR, Arnold PD. The role of glutamate signaling in the pathogenesis and treatment of obsessive-compulsive disorder. Pharmacol Biochem Behav 2012; 100: 726-35.

24 Rotge JY, Aouizerate B, Tignol J, Bioulac B, Burbaud P, Guehl D. The glutamate-based genetic immune hypothesis in obsessive-compulsive disorder. An integrative approach from genes to symptoms. Neuroscience 2010; 165: 408-17.

25 Najjar S, Pearlman DM, Alper K, Najjar A, Devinsky O. Neuroinflammation and psychiatric illness. J Neuroinflammation 2013; 10: 43.

26 Frick LR, Williams K, Pittenger C. Microglial dysregulation in psychiatric disease. Clin Dev Immunol 2013; 2013: 608-54.

27 Brimberg L, Benhar I, Mascaro-Blanco A, Alvarez K, Lotan D, Winter C, et al Behavioural, pharmacological, and immunological abnormalities after streptococcal exposure: a novel rat model of Sydenham chorea and related neuropsychiatric disorders. Neuropsychopharmacology 2013; 37: 2076-87.

28 Kirvan CA, Cox CJ, Swedo SE, Cunningham MW. Tubulin is a neuronal target of autoantibodies in Sydenham's chorea. J Immunol 2007; 178: 7412-21.

29 Kirvan CA, Swedo SE, Heuser JS, Cunningham MW. Mimicry and autoantibody-mediated neuronal cell signaling in Sydenham chorea. Nat Med 2003; 9: 914-20.

30 Kirvan CA, Swedo SE, Snider LA, Cunningham MW. Antibody-mediated neuronal cell signaling in behaviour and movement disorders. $J$ Neuroimmunol 2006; 179: 173-9.

31 Cunningham MW. Streptococcus and rheumatic fever. Curr Opin Rheumatol 2012; 24: 408-16.

32 Swedo SE, Leonard HL, Rapoport JL. The pediatric autoimmune neuropsychiatric disorders associated with streptococcal infection (PANDAS) subgroup: separating fact from fiction. Pediatrics 2004; 113: 907-11.

33 Swedo SE, Leonard HL, Garvey M, Mittleman B, Allen AJ, Perlmutter, et al. Pediatric autoimmune neuropsychiatric disorders associated with streptococcal infections: clinical description of the first 50 cases. Am Psychiatry 1998; 155: 264-71.

34 Singer HS, Giuliano JD, Hansen BH, Hallett JJ, Laurino JP, Benson M, et al. Antibodies against human putamen in children with Tourette syndrome. Neurology 1998; 50: 1618-24.

35 Kansy JW, Katsovich L, Mclver KS, Pick J, Zabriskie JB, Lombroso PJ, et al. Identification of pyruvate kinase as an antigen associated with Tourette syndrome. J Neuroimmunol 2006; 181: 165-76.

36 Fibbe LA, Cath DC, van den Heuvel OA, Veltman DJ, Tijssen MA, van Balkom AJ. Relationship between movement disorders and obsessive-compulsive disorder: beyond the obsessive-compulsive-tic phenotype. A systematic review. J Neurol Neurosurg Psychiatry 2012; 83: 646-54.

37 Hounie AG, Pauls DL, Mercadante MT, Rosario-Campos MC, Shavitt RD, de Mathis MA, et al. Obsessive-compulsive spectrum disorders in rheumatic fever with and without Sydenham's chorea. J Clin Psychiatry 2004; 65 994-9.

38 Maia DP, Teixeira Jr AL, Quintao Cunningham MC, Cardoso F. Obsessive compulsive behaviour, hyperactivity, and attention deficit disorder in Sydenham chorea. Neurology 2005; 64: 1799-801.

39 Mercadante MT, Diniz JB, Hounie AG, Ferrao Y, Alvarenga P, Brotto S, et al. Obsessive-compulsive spectrum disorders in rheumatic fever patients. J Neuropsychiatry Clin Neurosci 2005; 17: 544-7.

40 Asbahr FR, Garvey MA, Snider LA, Zanetta DM, Elkis H, Swedo SE. Obsessive-compulsive symptoms among patients with Sydenham chorea. Biol Psychiatry 2005; 57: 1073-6.
41 Swedo SE, Leonard HL, Casey BJ, Mannheim GB, Lenane MC, Rettew DC, et al. Sydenham's chorea: physical and psychological symptoms of St Vitus dance. Pediatrics 1993; 91: 706-13.

42 Martino D, Church A, Giovannoni G. Are antibasal ganglia antibodies important, and clinically useful? Pract Neurol 2007; 7: 32-41.

43 Kirvan CA, Swedo SE, Kurahara D, Cunningham MW. Streptococcal mimicry and antibody-mediated cell signaling in the pathogenesis of Sydenham's chorea. Autoimmunity 2006; 39: 21-9.

44 Dale RC, Brilot F. Autoimmune basal ganglia disorders. J Child Neurol 2012; 27: $1470-81$.

45 Dale RC, Church AJ, Surtees RA, Lees AJ, Adock JE, Harding B, et al. Encephalitis lethargica syndrome: 20 new cases and evidence of basal ganglia autoimmunity. Brain 2004; 127: 21-33.

46 Sanchez-Carpintero R, Albesa SA, Crespo N, Villoslada P, Narbona J. A preliminary study of the frequency of anti-basal ganglia antibodies and streptococcal infection in attention deficit/hyperactivity disorder. J Neurol 2009; 256: 1103-8.

47 Yaddanapudi K, Hornig M, Serge R, De Miranda J, Baghban A, Villar G, et al. Passive transfer of streptococcus-induced antibodies reproduces behavioural disturbances in a mouse model of pediatric autoimmune neuropsychiatric disorders associated with streptococcal infection. Mol Psychiatry 2010; 15 : 712-26.

48 Dale RC, Merheb V, Pillai S, Wang D, Cantrill L, Murphy TK, et al. Antibodies to surface dopamine-2 receptor in autoimmune movement and psychiatric disorders. Brain 2012; 135: 3453-68.

49 Dale RC, Candler PM, Church AJ, Wait R, Pocock JM, Giovannoni G. Neuronal surface glycolytic enzymes are autoantigen targets in post-streptococcal autoimmune CNS disease. J Neuroimmunol 2006; 172: 187-97.

50 Moher D, Liberati A, Tetzlaff J, Altman DG, Group P. Preferred reporting items for systematic reviews and meta-analyses: the PRISMA statement. Ann Intern Med 2009; 151: 264-9, W264.

51 Stroup DF, Berlin JA, Morton SC, Olkin I, Wilkinson GD, Rennie D, et al. Metaanalysis of observational studies in epidemiology: a proposal for reporting. Meta-analysis Of Observational Studies in Epidemiology (MOOSE) group. JAMA 2000; 283: 2008-12.

52 Wells G, Shea B, O'Connell D, Peterson J, Welch V, Losos M, et al. The Newcastle-Ottawa Scale (NOS) for assessing the quality of nonrandomised studies in meta-analyses. Presented at the 3rd Symposium on Systematic Reviews: Beyond the Basics, 3-5 July 2000 (http://www.ohri.ca/programs/ clinical_epidemiology/oxford.asp).

53 Fritschy JM. Is my antibody-staining specific? How to deal with pitfalls of immunohistochemistry. Eur J Neurosci 2008; 28: 2365-70.

54 Church AJ, Dale RC, Giovannoni G. Anti-basal ganglia antibodies: a possible diagnostic utility in idiopathic movement disorders? Arch Dis Child 2004; 89: 611-4.

55 DerSimonian R, Laird N. Meta-analysis in clinical trials. Control Clin Trials 1986; 7: 177-88

56 Higgins JP, Thompson SG, Deeks JJ, Altman DG. Measuring inconsistency in meta-analyses. BMJ 2003; 327: 557-60.

57 Egger M, Davey Smith G, Schneider M, Minder C. Bias in meta-analysis detected by a simple, graphical test. BMJ 1997; 315: 629-34.

58 Harbord RM, Higgins JPT. Meta-regression in Stata. Stata J 2008; 8: 493-519.

59 Goodman WK, Price LH, Rasmussen SA, Mazure C, Fleischmann RL, Hill CL, et al. The Yale-Brown Obsessive-Compulsive Scale. I. Development, use, and reliability. Arch Gen Psychiatry 1989; 46: 1006-11.

60 Kawikova I, Grady BPX, Tobiasova Z, Zhang Y, Vojdani A, Katsovich L, et al. Children with Tourette's syndrome may suffer immunoglobulin A dysgammaglobulinemia: preliminary report. Biol Psychiatry 2010; 67: 679-83.

61 Singer H, Gause C, Grados M. Antineuronal antibodies in obsessivecompulsive disorder: Comparisons in children with OCD-only, OCD + chronic TICS and OCD + PANDAS. Eur Psychiatry 2009; 24: S175.

62 Zuccolo A, Mascaro-Blanco A, Alvarez K, Stoner J, Cunningham M. Antineuronal antibodies in a heterogeneous 'PANDAS' group with tics and obsessive compulsive disorder. Dev Med Child Neurol 2012; 54: 141.

63 Murphy T, Storch E. The relationship between streptococcal antibodies and the course of OCD and tics in a pediatric population. Eur Psychiatry 2009; 24: S177.

64 Swedo SE, Kilpatrick K, Shapiro MB, Mannheim G, Leonard H. Antineuronal antibodies (AnA) in Sydenham's Chorea (SC) and obsessive-compulsive disorder (OCD) [abstract]. Pediatr Res 1991; 29: 364A.

65 Nicholson TRJ, Ferdinando S, Krishnaiah RB, Anhoury S, Lennox BR, Mataix-Cols $D$, et al. Prevalence of anti-basal ganglia antibodies in adult obsessive-compulsive disorder: cross-sectional study. Br J Psychiatry 2012 200: $381-6$ 
66 Gause C, Morris C, Vernekar S, Pardo-Villamizar C, Grados MA, Singer HS. Antineuronal antibodies in OCD: comparisons in children with OCD-only, OCD+chronic tics and OCD+PANDAS. J Neuroimmunol 2009; 214: 118-24.

67 Maina G, Albert U, Bogetto F, Borghese C, Cat Berro A, Mutani R, et al. Anti-brain antibodies in adult patients with obsessive-compulsive disorder. J Affect Disord 2009; 116: 192-200.

68 Bhattacharyya S, Khanna S, Chakrabarty K, Mahadevan A, Christopher R, Shankar SK. Anti-brain autoantibodies and altered excitatory neurotransmitters in obsessive-compulsive disorder. Neuropsychopharmacology 2009; 34: 2489-96.

69 Morer A, Lazaro L, Sabater L, Massana J, Castro J, Graus F. Antineurona antibodies in a group of children with obsessive-compulsive disorder and Tourette syndrome. J Psychiatr Res 2008; 42: 64-8.

70 Dale RC, Heyman I, Giovannoni G, Church AWJ. Incidence of anti-brain antibodies in children with obsessive-compulsive disorder. Br J Psychiatry 2005; 187: 314-9.

71 Trucco M, Leckman JF, Lombroso PJ. Autoantibodies and HLA-DR antigens in patients with TS, OCD and ADHD [abstract]. Presentation 580.24, Society for Neuroscience Annual Meeting 2000, New Orleans (available at http:// www.sfn.org/Annual-Meeting/Past-and-Future-Annual-Meetings/AbstractArchive).

72 Rose NR, Bona C. Defining criteria for autoimmune diseases (Witebsky's postulates revisited). Immunol Today 1993; 14: 426-30.

73 Witebsky E, Rose NR, Terplan K, Paine JR, Egan RW. Chronic thyroiditis and autoimmunization. JAMA 1957; 164: 1439-47.

74 Fluitman S, Denys D, Vulink N, Schutters S, Heijnen C, Westenberg H. Lipopolysaccharide-induced cytokine production in obsessive-compulsive disorder and generalized social anxiety disorder. Psychiatry Res 2010; 178 $313-6$

75 Fluitman SB, Denys DA, Heijnen CJ, Westenberg HG. Disgust affects TNFalpha, IL-6 and noradrenalin levels in patients with obsessive-compulsive disorder. Psychoneuroendocrinology 2010; 35: 906-11.

76 Poe LB. Radiological cases of the month. Gastrointestinal manifestations of hemolytic uremic syndrome. Am J Dis Child 1989; 143: 1481-2.

77 Rippel CA, Hong JJ, Yoon DY, Williams PN, Singer HS. Methodologic factors affect the measurement of anti-basal ganglia antibodies. Ann Clin Lab Sci 2005; 35: 121-30.

78 Morshed SA, Parveen S, Leckman JF, Mercadante MT, Bittencourt Kiss MH Miguel EC, et al. Antibodies against neural, nuclear, cytoskeletal, and streptococcal epitopes in children and adults with Tourette's syndrome, Sydenham's chorea, and autoimmune disorders. Biol Psychiatry 2001; 50: $566-77$

79 Dickerson F, Stallings C, Vaughan C, Origoni A, Khushalani S, Yolken R. Antibodies to the glutamate receptor in mania. Bipolar Disord 2012; 14: $547-53$

80 Dalmau J, Gleichman AJ, Hughes EG, Rossi JE, Peng X, Meizan L, et al. Anti-NMDA-receptor encephalitis: case series and analysis of the effects of antibodies. Lancet Neurol 2008; 27: 1091-8.

81 Irani SR, Bera K, Waters P, Zulliani L, Maxwell S, Zandi MS, et al. N-methyl-Daspartate antibody encephalitis: temporal progression of clinical and paraclinical observations in a predominantly non-paraneoplastic disorder of both sexes. Brain 2010; 133: 1655-67.

\section{poem}

\section{The Man with the Wooden Leg}

\section{Katherine Mansfield}

There was a man lived quite near us;

He had a wooden leg and a goldfinch in a green cage.

His name was Farkey Anderson,

And he'd been in a war to get his leg.

We were very sad about him,

Because he had such a beautiful smile

And was such a big man to live in a very small house.

When he walked on the road his leg did not matter so much;

But when he walked in his little house

It made an ugly noise.

Little Brother said his goldfinch sang the loudest of all birds,

So that he should not hear his poor leg

And feel too sorry about it.

Selected by Femi Oyebode. 